“ (C) 2011 IEEE. Personal use of this material is permitted. Permission from IEEE must be obtained for all other uses, in any current or future media, including

reprinting/republishing this material for advertising or promotional purposes, creating new collective works, for resale or redistribution to servers or lists, or reuse of any copyrighted component of this work in other works." 


\title{
Enhancing Frequency-Scanning Response of Leaky-Wave Antennas Using High Impedance Surfaces
}

\author{
María García-Vigueras, Student Member, IEEE, José Luis Gómez-Tornero, Member, IEEE, George
} Goussetis, Member, IEEE, Andrew R. Weily, Member, IEEE, and Y. Jay Guo, Senior Member, IEEE

\begin{abstract}
The use of High-Impedance Surfaces (HIS) to increase the frequency-scanning sensitivity of hollow leaky-wave antennas (LWAs) is presented. The LWA consists of a hollow rectangular waveguide with one of its narrow walls replaced by a Partially Reflective Surface (PRS) and it is loaded with a metallodielectric HIS. Theoretical results based on a simple Transverse Equivalent Network illustrate the physical mechanism responsible for the improvement, and they are verified by experiments on a prototype working in the $11 \mathrm{GHz}-16 \mathrm{GHz}$ band.
\end{abstract}

Index Terms-Leaky-wave antennas, frequency beam scanning, high impedance surfaces.

\section{INTRODUCTION}

$\mathrm{F}_{\mathrm{k}}$

REQUENCY-beam scanning is a well-known property of leaky-wave antennas (LWAs) [1]. Due to the dispersive nature of a leaky-mode complex propagation constant $k(f)$, the associated highly-directive main-beam elevation angle $\theta_{R A D}$ can be frequency scanned according to the following relations [1]:

$$
\begin{aligned}
& k(f)=\beta(f)-j \alpha(f) \\
& \theta_{R A D}(f) \approx \arcsin \frac{\beta(f)}{k_{0}}
\end{aligned}
$$

where $\beta$ is the leaky-mode phase constant, $\alpha$ is its leakage rate $k_{0}$ is the free-space wave number, and $\theta_{R A D}$ is measured from the broadside direction. LWAs offer an attractive mechanism to frequency scan the radiated beam of electrically large apertures by simply feeding a leaky-mode, thus avoiding more expensive feeding networks associated with array antennas. Frequency-scanned LWAs are also much simpler compared to electronically-scanned phased-arrays, which scan the radiated

Manuscript received May 30, 2010. This work has been supported by Spanish National project TEC2007-67630-C03-02/TCM, Regional Seneca project $08833 / \mathrm{PI} / 08$, and by Spanish scholarship "Salvador de Madariaga" project $08833 / \mathrm{PI} / 08$,
(ref. PR2009-0336)

(ref. PR2009-0336).
M. García-Vigueras and J.L. Gómez-Tornero are with the Department of Communication and Information Technologies, Technical University of Cartagena, Cartagena 30202 Spain (phone: +34968326531; e-mail: maria.garcia@upct.es, josel.gomez@upct.es). G. Goussetis is with the Institute of Electronics Communications and Information Technology, Queen's University Belfast, BT3 9DT, UK. A.R. Weily and Y.J. Guo are with the CSIRO ICT Centre, PO Box 76, Epping, NSW 1710, Australia. beam at a fixed frequency [2]. However, frequency scanned antennas may lead to higher front-end complexity due to the broader bandwidth needed to perform the scanning. Therefore, it is desirable to increase the antenna frequency sensitivity (i.e, how quickly the beam angle scans as the frequency is varied) in order to reduce the bandwidth of the circuitry associated with frequency-scanned LWAs.

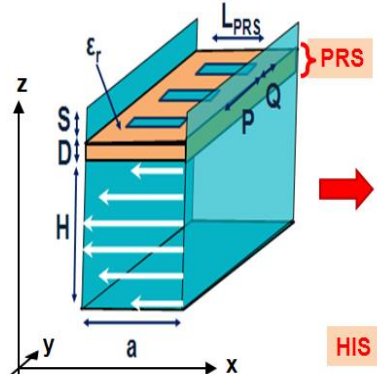

a)

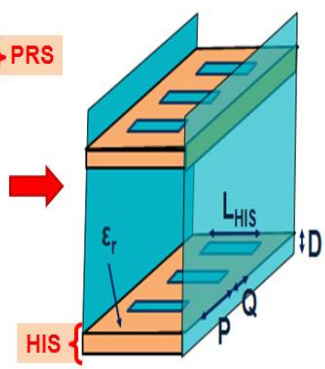

b)
Fig. 1. a) Hollow LWA and b) LWA loaded with HIS ( $a=H=11 \mathrm{~mm}, S=5 \mathrm{~mm}$, $D=1.13 \mathrm{~mm}, \epsilon_{r}=2.2$, LPRS $=10 \mathrm{~mm}$, LHIS $=9 \mathrm{~mm}, P=1.5 \mathrm{~mm}, Q=0.5 \mathrm{~mm}$ ).

The frequency-scan behavior of LWAs depends on the dispersive response of the associated leaky-mode phase constant $\beta(f)$, as stated by Eq.(2). Particularly, LWAs based on dielectric-filled waveguides present higher frequency sensitivity and higher range of scanned angles than LWAs based on hollow air-filled waveguides [3-5]. For a rectangular waveguide of height $H$ with relative permittivity $\varepsilon_{r}$ operating in the perturbed $\mathrm{TE}_{01}$ leaky-mode such as the one shown in Fig,1, and assuming small perturbation conditions, the lower and upper frequencies of operation for broadside $\left(\theta_{R A D}=0^{\circ}\right)$ and endfire $\left(\theta_{R A D}=90^{\circ}\right)$ scanning can be approximated by:

$$
\begin{aligned}
& f_{\text {BROADSIDE }} \approx \frac{c_{0}}{2 H} \frac{1}{\sqrt{\varepsilon_{r}}} \\
& f_{\text {ENDFIRE }} \approx \frac{c_{0}}{2 H} \frac{1}{\sqrt{\varepsilon_{r}-1}}=f_{\text {BROADSIDE }} \sqrt{\frac{\varepsilon_{r}}{\varepsilon_{r}-1}}
\end{aligned}
$$

As can be seen by (4), the bandwidth needed to scan from broadside to endfire is reduced as $\varepsilon_{r}$ is increased, while hollow waveguides $\left(\varepsilon_{r}=1\right)$ would require very large bandwidths to 
approach angles close to endfire, thus limiting the scanning range in practice. On the other hand, dielectric-filled LWAs have higher losses associated with the dielectric medium and they suffer from higher changes in the beamwidth as frequency is varied [1]. For these reasons, it would be desirable to create a LWA based on a hollow waveguide to minimize the ohmic losses and beamwidth dispersion, but with increased frequency sensitivity to perform the scanning in the minimum bandwidth With this main objective in mind, we present a new LWA based on a hollow rectangular waveguide with its top narrow wall replaced by a Partially Reflective Surface (PRS) (as described in [6]) by adding a High Impedance Surface (HIS) [7] in the bottom narrow wall, as illustrated in Fig.1. In Section II, the effect of the HIS on the leaky-mode frequencydispersion will be theoretically studied by using an efficient TEN (Transverse Equivalent Network) developed in [6], illustrating how the HIS increases the frequency sensitivity of the original hollow-waveguide LWA. Experimental results on manufactured prototypes are reported in Section III, validating the proposed mechanism to improve the LWA frequencyscanning response. Finally, Section IV presents the conclusions of this work.

\section{ANALYSIS OF HOLLOW LWA LOADED WITH HIS}

Figure 1-a shows a LWA based on a hollow rectangular waveguide with a metallodielectric PRS formed by printed dipoles [6]. The highly-reflective PRS allows the propagation of a $\mathrm{TE}_{01}$-type leaky-mode with a weak leakage rate, providing a large radiating length and therefore high directivity. The dispersion with frequency of this leaky-mode can be analyzed by using a simple but accurate TEN developed in [6]. The dimensions of the structure to operate in the $15 \mathrm{GHz}$ frequency band are also shown in Fig.1. Figure 2 shows how the pointing angle of this LWA is scanned from $\theta_{R A D}=5^{\circ}$ at $f=13 \mathrm{GHz}$ to $\theta_{R A D}=65^{\circ}$ at $f=26 \mathrm{GHz}$. If the waveguide is filled with a dielectric medium (for instance Teflon, with $\varepsilon_{r}=2.2$ ), and the cavity height $H$ is decreased from $H=11 \mathrm{~mm}$ to $H=9.5 \mathrm{~mm}$ in order to operate in a similar frequency band, a more sensitive scanning response is obtained, which is from $\theta_{R A D}=5^{\circ}$ at $f=11 \mathrm{GHz}$ to $\theta_{R A D}=90^{\circ}$ at $f=15 \mathrm{GHz}$, thus increasing the range of scanned angles up to endfire and reducing the bandwidth, in accordance with (3-4).

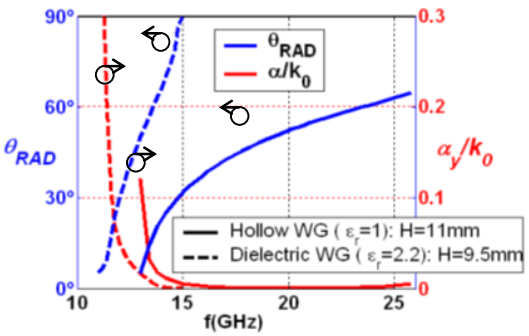

Fig. 2. Leaky-mode frequency dispersion for the LWA of Fig.1-a with and without dielectric filling to observe the change in the frequency-scanning.

To avoid the use of dielectric and its associated losses, a metallodielectric HIS formed by a grounded array of printed dipoles [6,7] can be added at the bottom wall of the hollow leaky waveguide as shown in Fig.1-b. The HIS strongly modifies the boundary conditions seen by the incident $\mathrm{TE}_{01}$ type mode[6]. Particularly, the length of the HIS dipoles ( $L_{H I S}$ in Fig.1-b) can be designed to create an Artificial Magnetic Conductor (AMC) condition which ultimately causes the leaky-mode to enter a surface-wave regime $\left(\beta / k_{0}>1\right)$ [6], thus increasing the LWA frequency scanning sensitivity up to endfire. This fact is shown in Fig.3-a, where theoretical leakymode frequency dispersion results obtained using the TEN are given for different illustrative scenarios (without HIS and with $L_{H I S}=5 \mathrm{~mm}$ and $L_{H I S}=5.5 \mathrm{~mm}$ ).
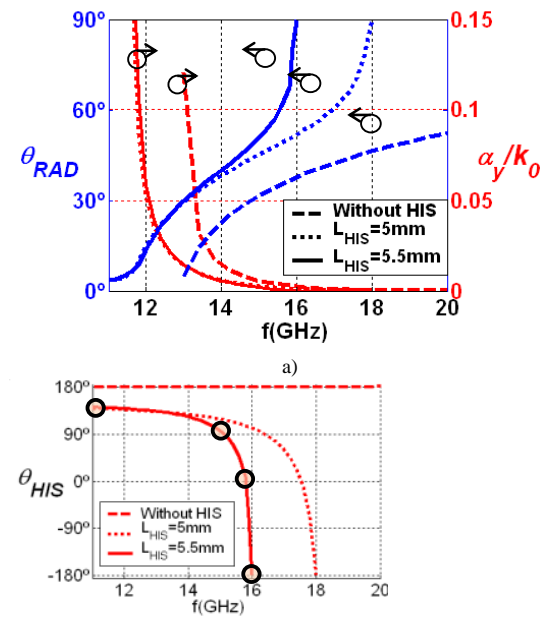

b)

Fig. 3. a) Leaky-mode frequency dispersion for the hollow LWA of Fig.1 with different HIS dipoles length b) Reflection phase presented by the HIS.

As shown in Fig.3-a, the beam-scanning curve becomes more sensitive to frequency change when the HIS is present. Moreover, by properly tuning the length of the HIS dipoles $L_{H I S}$ [6], one can increase this sensitivity further. To understand this effect, we adopt the bouncing plane wave interpretation for waveguide propagation [9] and plot the reflection phase, $\theta_{H I S}$, experienced by the wave travelling along the leaky waveguide at the HIS plane. Fig.3-b plots $\theta_{H I S}$ versus frequency for the different scenarios of Fig.3-a. In the absence of the HIS, the boundary condition at this plane corresponds to a metallic wall and the reflection phase has a constant value of $+180^{\circ}$ for all frequencies and associated scanned angles $\theta_{R A D}$. When the HIS is introduced, the reflection phase experienced at this boundary, $\theta_{H I S}$, rapidly changes with frequency from $\theta_{H I S}=+140^{\circ}$ to $\theta_{H I S}=-180^{\circ}$, as shown in Fig.3-b. Particularly, the AMC condition $\left(\theta_{H I S}=0^{\circ}\right)$ can be tuned to lower or higher frequencies by modifying the 
HIS dipoles length $L_{H I S}$ [7]. As presented in Fig.3-b, the AMC condition is set to $17.5 \mathrm{GHz}$ for $L_{H I S}=5 \mathrm{~mm}$ and to $15.8 \mathrm{GHz}$ for $L_{H I S}=5.5 \mathrm{~mm}$. For clarity we note that the angle of incidence of the bouncing wave at the HIS plane, which is equal to the pointing angle of the radiated beam, $\theta_{R A D}$, at these points is $68.5^{\circ}$ and $67^{\circ}$ respectively. This AMC resonance condition establishes the frequency in which the leaky-mode pointing angle $\theta_{R A D}$ is pushed to high values (around $\theta_{R A D}=70^{\circ}$ in Fig.3a), eventually obtaining the endfire direction $\left(\theta_{R A D}=90^{\circ}\right)$ when frequency is increased, and finally entering the surface-wave regime $\left(\beta / k_{0}>l\right.$ with an imaginary value of $\left.\theta_{R A D}[1]\right)$. In this way, by tuning the AMC resonance one can make the endfire radiation frequency to be located close to the broadside radiation frequency (which is around $11 \mathrm{GHz}$ for the HISloaded LWAs, as can be seen in Fig.3-a), hence reducing the bandwidth needed to scan the beam. This is the reason why the broadside to endfire frequency range is reduced to $[11 \mathrm{GHz}$ $18 \mathrm{GHz}$ ] when $L_{H I S}=5 \mathrm{~mm}$, and to $[11 \mathrm{GHz}-16 \mathrm{GHz}]$ when $L_{H I S}=5.5 \mathrm{~mm}$ (see Fig.3-b).

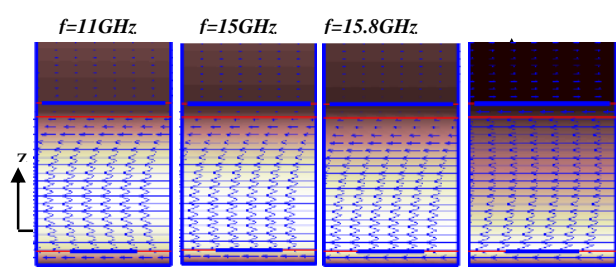

Fig. 4. Leaky-mode electric field distribution inside the HIS-loaded LWA for different frequencies $($ LHIS $=5.5 \mathrm{~mm})$.

For the case $L_{H I S}=5.5 \mathrm{~mm}$, Fig. 4 presents the leaky-mode electric field inside the LWA cavity at four frequencies of the scanning range. The related values of $\theta_{H I S}$ are highlighted with circles in Fig.3-b. At $11 \mathrm{GHz}$, the HIS behaves approximately as a grounded dielectric slab $\left(\theta_{H I S}=+140^{\circ}\right.$ in Fig.3-b) and a perturbation of the usual waveguide $\mathrm{TE}_{10}$ mode occurs in the LWA cavity (the cavity height $H$ corresponds to halfwavelength of the resonant fields in the transverse direction, see Fig.4). The hollow cavity height $H$ is designed using (3) to provide near broadside radiation (waveguide close to cutoff) at this frequency $\left(\theta_{R A D} \approx 0^{\circ}\right.$ in Fig.3-a). The effect of the HIS starts to appear as frequency is increased, rapidly decreasing $\theta_{H I S}$ and increasing $\theta_{R A D}$ due to the AMC resonance as shown in Fig.3. At $15 \mathrm{GHz}, \theta_{R A D}$ has been increased to $+48^{\circ}$. At $15.8 \mathrm{GHz}$, the HIS presents AMC resonance condition $\left(\theta_{H I S}=O^{\circ}\right.$ in Fig.3-b) which manifests in maximum average tangential electric fields intensity at the HIS interface (Fig.4). As a result of the AMC condition the effective cavity height is increased [6], making the scanned angle rise suddenly $\left(\theta_{R A D} \approx 70^{\circ}\right.$ at $15.8 \mathrm{GHz}$ in Fig.3-a) with respect to the case without HIS $\left(\theta_{R A D} \approx 40^{\circ}\right.$ in Fig.3-a). When frequency is augmented from this point, the leaky-mode passes by the endfire condition $\left(\theta_{R A D} \approx 90^{\circ}\right.$ at $16 \mathrm{GHz}$ in Fig.3-a) and eventually transforms into a surface-wave (which does not radiate). As illustrated in
Fig.4, the field is confined in the AMC dielectric slab at $16 \mathrm{GHz}$ with exponentially decreasing amplitude in the airfilled cavity region, as it corresponds to a surface-wave [1]

\section{EXPERIMENTAL RESULTS}

To experimentally confirm the effect of the HIS in the improvement of the leaky frequency-scanning response, three hollow LWAs were fabricated according to the dimensions of Fig.1. The first of them does not use any HIS, while the other two LWAs were loaded with metallodielectric HIS with $L_{H I S}=5 \mathrm{~mm}$ and $L_{H I S}=5.5 \mathrm{~mm}$, respectively. A picture of the manufactured prototype is shown in Fig.5. Figure 6 shows the frequency-scanning curves of the three manufactured LWAs, measured in the $10 \mathrm{GHz}-20 \mathrm{GHz}$ frequency range. The leakymode results from $10 \mathrm{GHz}$ to $26 \mathrm{GHz}$ obtained from the TEN are superimposed for comparison, showing good agreement between theory and experiments, and thus confirming the mechanism to enhance the scanning sensitivity and the range of scanned angles.

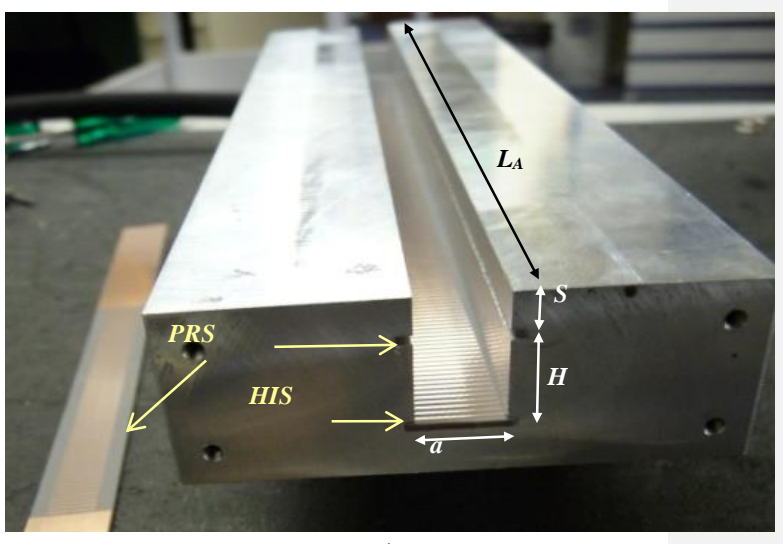

a)

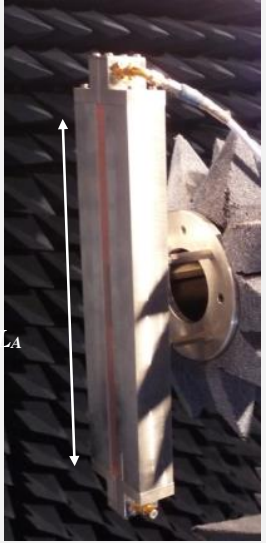

b)
Fig. 5. Photograph of manufactured LWA prototype, a) Metallic waveguide hosting the printed-circuit dipole-based PRS (ready to be inserted at the top) and HIS (at the bottom) b) Whole LWA structure at the anechoic chamber.

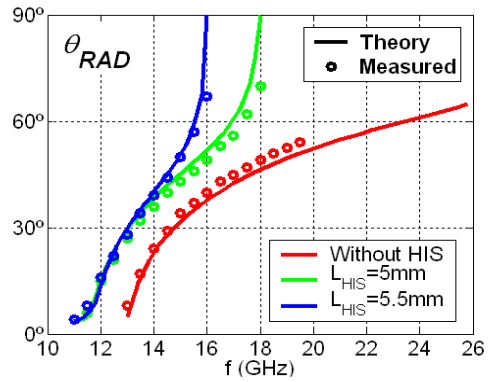

Fig. 6. Theoretical and measured frequency-scanning response of hollow LWAs showing the effect of the HIS. 
Fig.6 shows that the HIS-loaded LWA with $L_{H I S}=5.5 \mathrm{~mm}$ provides beam scanning from $\theta_{R A D}=4^{\circ}$ to $\theta_{R A D}=67^{\circ}$ in the frequency range $11 \mathrm{GHz}-16 \mathrm{GHz}$, while the LWA without HIS has a poorer frequency sensitivity (from near broadside to $\theta_{R A D}=65^{\circ}$ in the frequency range $13 G \mathrm{~Hz}-26 \mathrm{GHz}$ ). The theoretical and measured normalized radiation patterns for the HIS-loaded LWA with $L_{H I S}=5.5 \mathrm{~mm}$ and a radiating length $L_{A}=200 \mathrm{~mm}\left(9.2 \lambda_{0}\right.$ at $\left.14 \mathrm{GHz}\right)$ are plotted in Fig.7-a and Fig.7$\mathrm{b}$, respectively. Good agreement between the theoretical and measured radiation patterns is observed, showing the scanning of the main beam elevation angle $\theta_{R A D}$. The beamwidth broadens close to broadside $(f=11 \mathrm{GHz}$ in Fig.7) due to the increase of the leakage rate (see $\alpha / k_{0}$ curve in Fig.3-a fo $L_{H I S}=5.5 \mathrm{~mm}$ and $f=11 \mathrm{GHz}$ ) [1]. From $12 \mathrm{GHz}$ to $15 \mathrm{GHz}$, the expected frequency-beam steering is obtained. At $f=16 \mathrm{GHz}$, secondary lobe appears in the angle $\theta \approx 67^{\circ}$ as a result of the decrease in the leakage rate (see $\alpha / k_{0}$ curve in Fig.3-a for $L_{H I S}=5.5 \mathrm{~mm}$ and $f=16 \mathrm{GHz}$ ), which reduces the LWA radiation efficiency and creates a reflected leaky-wave pointing at a mirrored angle with respect to the main beam [1]. Radiation to pointing angles higher than $+70^{\circ}$ is limited by the appearance of grating lobes due to higher-order modes $[1,2,8]$. The measured insertion ohmic losses of the fabricated hollow LWA loaded with HIS is in the range of $1.5 \mathrm{~dB}$ for the entire frequency band, while for the case of a similar LWA based on a waveguide filled with Teflon $\left(\varepsilon_{r}=2.2, \tan \delta=0.005\right)$ the insertion losses are in the order of $6 d B$, as demonstrated in [3].
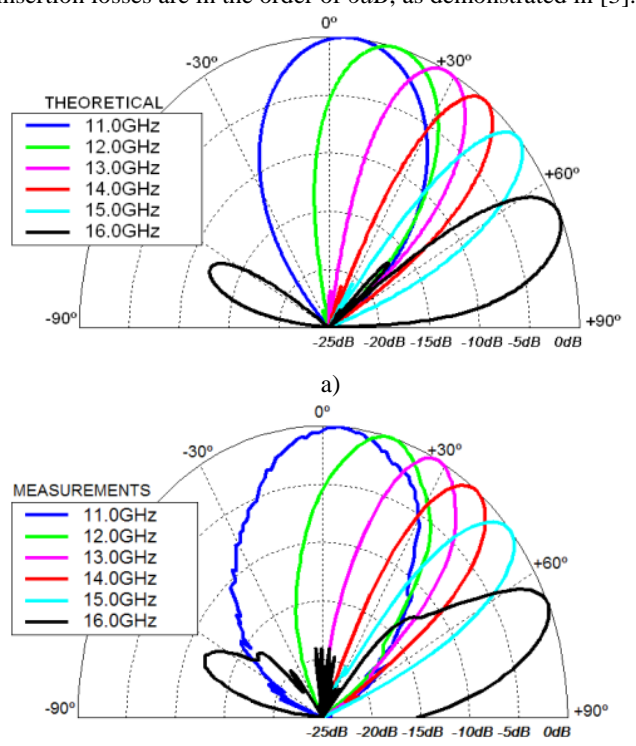

b)

Fig. 7. a) Theoretical and b) measured radiation diagram at differen frequencies to show the frequency beam-scanning of the designed HIS-loaded LWA $($ LHIS $=5.5 \mathrm{~mm})$.

\section{CONCLUSION}

This work has demonstrated the improvement in the frequency scanning sensitivity of a hollow LWA due to the insertion of a HIS. The new topology increases the frequency scanning sensitivity, thus reducing the required bandwidth to scan the main beam from near broadside to near endfire. It avoids the use of dielectric-filled waveguides, thus minimizing the associated dielectric losses. Theoretical results of the leaky-mode dispersion with frequency have been obtained from an efficient Transverse Equivalent Network to illustrate the physical mechanism. The HIS must be designed to introduce an AMC resonance condition close to the cut-off of the leaky-mode, so that the broadside and the endfire radiation frequencies are located in close proximity. Experimental results on fabricated prototypes have also been reported, showing very good agreement with theory. The designed HISloaded LWA shows a scanning range of $\left[5^{\circ}, 67^{\circ}\right]$ in a bandwidth from $11 \mathrm{GHz}$ to $16 \mathrm{GHz}$, achieving a frequency sensitivity of $12.4^{\circ} / \mathrm{GHz}$. A similar LWA without HIS presents a scanning range of $\left[7^{\circ}, 65^{\circ}\right]$ in the frequency range from $13 \mathrm{GHz}$ to $26 \mathrm{GHz}$, which has a frequency sensitivity of $4.5 \% \mathrm{GHz}$. Therefore, the frequency sensitivity is almost three times higher when the HIS is added to the hollow LWA. A dielectric-filled LWA with similar frequency sensitivity as the HIS-loaded air-filled LWA would introduce $4 d B$ higher insertion losses, subsequently decreasing the LWA radiation efficiency.

It should be noted that the frequency-scanning enhancement shown in this work can be extended to any type of air-filled LWAs (see examples in $[1,4,5]$ ) due to the fact that the HIS is able to perturb the leaky-mode by introducing a strongly dispersive AMC boundary condition

\section{REFERENCES}

[1] A.A. Oliner, "Leaky-wave antennas", in Antenna Engineering Handbook, 3rd ed, R.C. Johnson, Ed. New York, McGraw-Hill, 1993, Handbo 10.
Ch. 10.

[2] R.C. Hansen, Phased Array Antennas, John Wiley \& Sons, New York, 1998.

[3] J.L. Gómez, G.Goussetis, A.Feresidis, and A.A.Melcón, "Control of leaky-mode propagation and radiation properties in hybrid dielectricwaveguide printed-circuit technology: experimental results", IEEE Trans. Antennas Propagat., vol.54, No.11, pp.3383-3390, Nov. 2006.

[4] P. Lampariello, F. Frezza, H. Shigesawa, M. Tsuji and A.A. Oliner, "A versatile leaky-wave antenna based on stub-loaded rectangular waveguide: Part III-comparisons with measurements," IEEE Trans. Antennas Propagat, vol.46, no.7, pp.1047-1055, July 1998.

[5] M. Tsuji, T. Harada, H. Deguchi, and H. Shigesawa, "Frequencyscanning antennas with low sidelobes using stub-loaded ridgerectangular leaky waveguides," 2003 IEEE Topical Conference on Wireless Communication Technology, pp.352-353, 2003.

[6] M. García-Vigueras, J.L. Gómez-Tornero, G. Goussetis, J.S. GómezDiaz, and A. Álvarez-Melcón, "A modified pole-zero technique for the synthesis of waveguide leaky-wave antennas loaded with dipole-based FSS", IEEE Trans. Antennas Propag., in press.

[7] G. Goussetis, A.P. Feresidis, J.C. Vardaxoglou, "Tailoring the AMC and EBG Characteristics of periodic metallic arrays printed on grounded dielectric substrate," IEEE Trans. Antennas Propag, vol. 54, No. 1, pp. 82-89, Jan. 2006.
Commented [guo013 1]: I don't think the rest is necessary as we are ending the paper. 
[8] T. Zhao, D. R. Jackson, J. T. Williams, and A. A. Oliner, "General formulas for 2-D leaky-wave antennas," IEEE Trans. Antennas and Propag., vol. 53, No. 11, pp. 3515-3524, Nov. 2005.

[9] G. Goussetis, J.L Gómez-Tornero, A.P. Feresidis, and N. Uzunoglu, "Artificial impedance surfaces for reduced dispersion in antenna feeding systems", IEEE Transactions Antennas and Propagation, in press. 